\title{
Anomalous Transport and Nonlinear Reactions in Spiny Dendrites
}

\author{
Sergei Fedotov ${ }^{1}$, Hamed Al-Shamsi ${ }^{1}$, Alexey Ivanov ${ }^{2}$ and Andrey Zubarev ${ }^{2}$ \\ ${ }^{1}$ School of Mathematics, The University of Manchester, Manchester M60 1QD, UK and \\ 2 Department of Mathematical Physics, Ural State University, Russia
}

(Dated: September 10, 2018)

\begin{abstract}
We present a mesoscopic description of the anomalous transport and reactions of particles in spiny dendrites. As a starting point we use two-state Markovian model with the transition probabilities depending on residence time variable. The main assumption is that the longer a particle survives inside spine, the smaller becomes the transition probability from spine to dendrite. We extend a linear model presented in [PRL, 101, 218102 (2008)] and derive the nonlinear Master equations for the average densities of particles inside spines and parent dendrite by eliminating residence time variable. We show that the flux of particles between spines and parent dendrite is not local in time and space. In particular the average flux of particles from a population of spines through spines necks into parent dendrite depends on chemical reactions in spines. This memory effect means that one can not separate the exchange flux of particles and the chemical reactions inside spines. This phenomenon does not exist in the Markovian case. The flux of particles from dendrite to spines is found to depend on the transport process inside dendrite. We show that if the particles inside a dendrite have constant velocity, the mean particle's position $\langle x(t)\rangle$ increases as $t^{\mu}$ with $\mu<1$ (anomalous convection). We derive a fractional convection-diffusion equation for the total density of particles.
\end{abstract}

PACS numbers:

\section{INTRODUCTION}

Dendritic spines play a very important role in regulating neuronal activity of the cerebellar cortex because the majority of excitatory synapses are located on spines [1, 2]. The later are tiny bulbous protrusions from dendrites consisting of head $(\sim 1 \mu \mathrm{m})$ and thin neck $(\sim 0.1 \mu \mathrm{m})$. One of the main functions of spines is to help transmit electrical signals. The problem of propagation of action potential in the spiny dendrites has attracted enormous attention in past years. Several cable models have been suggested in the literature to study the spine-dendrite interaction on the macroscopic level. Baer and Rinzel [3] developed a phenomenological cable theory for spiny dendrites and found that the propagation rate of local excitation strongly depends on a spine-stem resistance. The dynamic structure of spines (the changes in the shape and size) has been studied in [4]. Modified FitzHugh-Nagumo model [5] was the subject of research by Coombes and Bressloff. Population of spines can be treated as a continuous function of spatial position along the dendrite [6, 7]. It should be noted that these cable models are phenomenological and not derived from the electro-diffusion equations for ions in spiny dendrites.

Recent experiments together with numerical simulations by Santamaria et al 8] showed that the transport of inert particles in spiny dendrites is anomalous. It was found that the mean-square displacement $\left\langle x^{2}(t)\right\rangle$ is proportional to $t^{\mu}$ with $\mu<1$. It turns out the dendritic spines act as traps of particles. The narrow spine neck significantly decreases the effective diffusion of particles into and out of dendritic spines. This results in slow anomalous diffusion along the shaft of dendrite [9]. Based on these experiments, Henry et al [10] suggested the fractional Nernst-Planck equations for electro-diffusion of ions in spiny dendrites and developed a cable model involving time-fractional derivatives. In particular, the average flow of ions between the shaft of dendrite and the spines is not local in time. Note that the coupling between spines and dendrites has been studied either phenomenologically or on microscopic level of a single spine [11 13]. The influence of a dendritic spine on the spread of calcium in the parent dendrite was studied in [14]. The passive diffusion in a tube with dead ends has been considered in [15]. Recently mesoscopic non-Markovian model for spines-dendrite interaction has been developed [16, 17]. The aim of this paper is to give an alternative Markovian model involving the residence time variable. This paper is an essential extension of [16] with new results and examples.

We are concerned with a mesoscopic description of the anomalous transport and reactions of particles in spiny dendrites. The mesoscopic approach involves a detailed description of the behavior of particles on the microscopic level. At the same time within mesoscopic approach we can introduce the mean densities of particles and neglect the random fluctuations around the mean behavior [17]. The system of linear Master equations for the mean density of particles inside a dendrite, $n_{1}(x, t)$, and the density of particles inside a population of spines, $n_{2}(x, t)$ has been derived in [16]. The starting point was non-Markovian continuous time random walk (CTRW) model involving the integral balance equations for average densities. The aim of this paper is to extend this analysis for non-linear case and derive a mesoscopic system of equations from Markovian model. One way to deal with non-Markovian process is 
to introduce supplementary variables that make the process Markovian [18, 19]. Here we introduce the residence time variable $\tau$ which is the time interval between arrival of particle in dendrite or spine and the time of leaving them. The particle has a zero residence time when it just arrives inside the spines from dendrite or inside dendrite from spines. This idea has been used in [18, 20 22].

\section{TWO-STATE REACTION-TRANSPORT MODEL}

We start with the microscopic two-state model of a random particle's movement in a spiny dendrite. When the particle is inside a dendrite, it performs a random walk for a random time $T_{1}$ (dendrite's residence time) before hitting the neck of spine. When the particle hits the neck, it is trapped inside the spine for a random time $T_{2}$ (spine's residence time). After spending the random time $T_{2}$ inside spine, the particle is released to the parent dendrite through spine neck. The particle starts to perform a random walk inside the dendrite until it hits the spine's neck again. Clearly the essential feature of this process can be described by two-state random process. The particle can be in one of the two states: inside dendrite or inside spines. We assume that the residence times or waiting times $T_{1}$ and $T_{2}$ are non-negative random variables with probability density functions (pdf's) $\psi_{1}(\tau)$ and $\psi_{2}(\tau)$ respectively. Note that the introduction of the residence time pdf's can be viewed as the probabilistic method to model the variability in the shape of dendritic spines and their density on the parent dendrite. The corresponding survival functions $\Psi_{i}(\tau)$ are defined as

$$
\Psi_{i}(\tau)=\int_{\tau}^{\infty} \psi_{i}(s) d s \quad i=1,2 .
$$

To describe the switching process we introduce two transition probabilities (hazard functions) [18]:

$$
\gamma_{i}(\tau)=\frac{\psi_{i}(\tau)}{\Psi_{i}(\tau)}, \quad i=1,2
$$

Thus $\gamma_{1}(\tau) h$ represents the conditional probability of transition from dendrite to spine in the small interval $(\tau, \tau+h)$ given that there is no transition up to time $\tau$. The product $\gamma_{2}(\tau) h$ has a similar meaning for the transition from spine

to dendrite. We also assume that inside spines the irreversible chemical reaction $C \stackrel{r_{2}^{-}}{\rightarrow} C_{b}$ with the rate $r_{2}^{-}$takes place. It describes the removal of the particles by immobile buffers and pumps [11, 12].

If we assume that the transition probabilities $\gamma_{1}(\tau)$ and $\gamma_{2}(\tau)$ are constants, then we have a classical two-state Markovian model. Master equations for the mean density of particles inside a dendrite, $n_{1}(x, t)$, and the density of particles inside a population of spines, $n_{2}(x, t)$, are

$$
\begin{gathered}
\frac{\partial n_{1}}{\partial t}=L_{x} n_{1}-\gamma_{1} n_{1}+\gamma_{2} n_{2} \\
\frac{\partial n_{2}}{\partial t}=-r_{2}^{-}\left(n_{2}\right) n_{2}-\gamma_{2} n_{2}+\gamma_{1} n_{1}
\end{gathered}
$$

where the reaction rate $r_{2}^{-}\left(n_{2}\right)$ depends on the local density of particles $n_{2}$ [17]. Here $L_{x}$ is the transport operator acting on $x$-coordinate along the dendrite.

To define the transport operator $L_{x}$, one can use the continuous-time random walk (CTRW) model in which the particles follow the path of the compound Poisson process. The probability of a jump during time interval of length $h$ is $\lambda(x) h+o(h)$ and jumps density function is $w(z)$ [17]. Then

$$
L_{x} n_{1}=--\lambda(x) n_{1}+\int_{\mathbb{R}} \lambda(z) n_{1}(z, t) w(x-z) d z .
$$

If we adopt the Nernst-Planck equation for the particles flow then

$$
L_{x} n_{1}=-\frac{\partial\left(v(x, t) n_{1}\right)}{\partial x}+D \frac{\partial^{2} n_{1}}{\partial x^{2}},
$$

where $D$ is the diffusivity of the particles. The advection velocity $v(x, t)=-\mu \partial \phi / \partial x$, where $\mu$ is the mobility, and the electrostatic potential $\phi$. Note that $v(x, t)$ depends on the total concentration of particles (ions) through the Poisson equation.

Our aim now is to extend the Markovian model (3) and (4) to the two-state semi-Markov model [17]. Applications of the semi-Markov processes to chemical kinetics can be found in [23]. Note that stochastic two-state models with nonexponential waiting time distributions occur in many areas of natural sciences. We mention the stochastic resonance theory [24], two-state model for anomalous diffusion [25], two-state gating process for ion channels [26], propagation of tumor cells [27], superdiffusion theory and random walk with memory [28]. 


\section{MARKOVIAN MODEL INVOLVING A RESIDENCE TIME VARIABLE}

The purpose of this section is to formulate the Markovian model for the transport and reactions of particles inside spiny dendrites. If the transition probabilities $\gamma_{1}(\tau)$ and $\gamma_{2}(\tau)$ are not constants, it is convenient to introduce the densities of particles depending on $\tau$ 20]. Let $\xi_{1}(x, \tau, t)$ be the density of particles at point $x$ at time $t$ whose residence time inside the dendrite lies in the interval $(\tau, \tau+d \tau)$. The corresponding density of particles inside a population of spines is $\xi_{2}(x, \tau, t)$. Integration of $\xi_{i}(x, \tau, t)$ over residence time variable $\tau$ gives the mean densities $n_{i}(t, x)$ at point $x$ at time $t$ :

$$
n_{i}(x, t)=\int_{0}^{\infty} \xi_{i}(x, \tau, t) d \tau, \quad i=1,2 .
$$

This model is similar to well-known age-structured models in which the population density of individuals depends explicitly on the age $\tau$ [31]. Of course, we should make a clear distinction between the residence time $\tau$ since the last jump and the residence time of a particle from $t=0$. The latter is not considered here.

The crucial question here whether the particles "remember" how long they have been inside spines or dendrite. The experiments [8] suggest the existence of the memory effects which lead to the anomalous transport of particles along the dendrite. To model these effects, we assume that the transition probabilities $\gamma_{i}(\tau)$ depend on the residence time variable $\tau$ [18]. Thus the probability of a transition from a dendrite to spines during a small time interval of length $h$ is $\gamma_{1}(\tau) h+o(h)$, and the backward transition has the probability $\gamma_{2}(\tau) h+o(h)$.

Since the average movement of particles inside the dendrite is governed by the operator $L_{x}$, the balance equations for $\xi_{1}(x, \tau, t)$ and $\xi_{2}(x, \tau, t)$ are

For a dendrite

$$
\frac{\partial \xi_{1}}{\partial t}+\frac{\partial \xi_{1}}{\partial \tau}=L_{x} \xi_{1}-\gamma_{1}(\tau) \xi_{1}
$$

For spines

$$
\frac{\partial \xi_{2}}{\partial t}+\frac{\partial \xi_{2}}{\partial \tau}=-\gamma_{2}(\tau) \xi_{2}-r_{2}^{-}\left(n_{2}\right) \xi_{2}
$$

The derivation of (8) and (9) together with the transport operator (5) is given in Appendix A. Note that this Markovian model can be easily generalized to include various nonlinear terms.

Initial conditions are

$$
\xi_{i}(x, \tau, 0)=n_{i}^{0}(x) f_{i}(\tau \mid x) \quad i=1,2
$$

where $n_{i}^{0}(x)$ is the initial densities of particles and $f_{i}(\tau \mid x)$ is the conditional waiting time distribution for the particles inside dendrite $(i=1)$ and spines $(i=2)$ at time $t=0$ with the property $\int_{0}^{\infty} f_{i}(\tau \mid x) d \tau=1$ [20, 21] .

Boundary conditions at $\tau=0$ :

For a dendrite

$$
\xi_{1}(x, 0, t)=\int_{0}^{\infty} \gamma_{2}(\tau) \xi_{2}(x, \tau, t) d \tau
$$

For spines

$$
\xi_{2}(x, 0, t)=\int_{0}^{\infty} \gamma_{1}(\tau) \xi_{1}(x, \tau, t) d \tau
$$

These boundary conditions have the following meaning. Particles inside dendrite with the residence time $\tau=0$ at the point $x$ are created with the rate $\gamma_{2}(\tau)$ (see (11)). The density of particles just arriving inside dendrite, $\xi_{1}(x, 0, t)$, can be found by integration of the product $\gamma_{2}(\tau) \xi_{2}(x, \tau, t)$ over all residence times. The density of particles just arriving inside spines $\xi_{2}(x, 0, t)$ can be found in a similar way (see (12)).

Now we assume that the convective velocity $v(x, t)=v=$ const and the jump rate $\lambda(x)=$ const. Using the method of characteristics and the Fourier transform, we obtain the solutions to (8) and (9) (see Appendix B):

For a dendrite

$$
\left.\xi_{1}(x, \tau, t)=e^{-\int_{0}^{\tau} \gamma_{1}(s) d s} \int_{\mathbb{R}} \xi_{1}(z, 0, t-\tau) p(x-z, \tau)\right) d z \quad \tau<t
$$




$$
\left.\xi_{1}(x, \tau, t)=e^{-\int_{\tau-t}^{\tau} \gamma_{1}(s) d s} \int_{\mathbb{R}} \xi_{1}(z, \tau-t, 0) p(x-z, t)\right) d z \quad \tau>t .
$$

For spines

$$
\begin{array}{ll}
\xi_{2}(x, \tau, t)=\xi_{2}(x, 0, t-\tau) e^{-\int_{0}^{\tau} \gamma_{2}(s) d s-\int_{t-\tau}^{\tau} r_{2}^{-}\left(n_{2}(x, s)\right) d s} & \tau<t, \\
\xi_{2}(x, \tau, t)=\xi_{2}(x, \tau-t, 0) e^{-\int_{\tau-t}^{\tau} \gamma_{2}(s) d s-\int_{0}^{t} r_{2}^{-}\left(n_{2}(x, s)\right) d s} & \tau>t .
\end{array}
$$

Here $p(x, t)$ is the Green function for the transport equation

$$
\frac{\partial p}{\partial t}=L_{x} p
$$

with the initial condition

$$
p(x, 0)=\delta(x)
$$

In particular, when the advection velocity $v=$ const in (6) we have

$$
p(x, t)=\frac{1}{\sqrt{4 \pi D t}} \exp \left[-\frac{(x-v t)^{2}}{4 D t}\right] .
$$

Note that (8) or (9) has different solutions for $\tau<t$ and $\tau>t$. When $\tau<t$, the solution (13) describes the evolution of particles that arrived inside dendrite from spines after $t=0$. The formula (14) gives the evolution of density of those particles that were present inside dendrite at $t=0$.

The formulas (13) and (14) can be generalized for the case when the advection velocity $v$ and jump rate $\lambda$ depend on space and time. We can write

$$
\begin{array}{ll}
\left.\xi_{1}(x, \tau, t)=e^{-\int_{0}^{\tau} \gamma_{1}(s) d s} \int_{\mathbb{R}} \xi_{1}(z, 0, t-\tau) p(x, \tau \mid z)\right) d z & \tau<t, \\
\left.\xi_{1}(x, \tau, t)=e^{-\int_{\tau-t}^{\tau} \gamma_{1}(s) d s} \int_{\mathbb{R}} \xi_{1}(z, \tau-t, 0) p(x, t \mid z)\right) d z & \tau>t,
\end{array}
$$

where the Green function $p(x, t \mid z)$ can be interpreted as the probability density function for a particle which starts at point $z$ in a dendrite and arrives at point $x$ at time $t$ without trapping in spines up to time $t$. The pdf $p(x, t \mid z)$ obeys the equation $\partial p / \partial t=L_{x} p$ with the initial condition $p(x, 0 \mid z)=\delta(x-z)$. Of course for nonhomogeneous case such as (5]) or (6) the explicit expression for $p(x, t \mid z)$ is not available.

The density $\xi_{i}(x, \tau, t)$ can be also interpreted as the probability density function of finding the particle inside the dendrite $(i=1)$ or spines $(i=2)$ at the point $x$ at time $t$ such that the residence time lies in the interval $(\tau, \tau+d \tau)$. Since $\dot{\Psi}_{i}(\tau)=\psi_{i}(\tau)$, it follows from (2) that the survival function $\Psi_{i}(\tau)$ is

$$
\Psi_{i}(\tau)=e^{-\int_{0}^{\tau} \gamma_{i}(s) d s} \quad i=1,2 .
$$

One can see that this exponential factor appears in the solutions (13)-(16). That is why (13)-(16) have a very simple probabilistic meaning of the law of total probability. Note that the residence time's density $\psi_{i}(\tau)$ can be written in terms of the transition rate $\gamma_{i}(\tau)$ as follows [18]

$$
\psi_{i}(\tau)=\gamma_{i}(\tau) e^{-\int_{0}^{\tau} \gamma_{i}(s) d s} \quad i=1,2
$$

It is natural to assume that the longer a particle survives inside spine, the smaller becomes the transition probability from spine to dendrite. In this case the transition rate $\gamma_{2}(\tau)$ is a monotonically decreasing function of residence time $\tau$. For example, if

$$
\gamma_{2}(\tau)=\frac{\mu}{\beta+\tau}
$$

then the survival function

$$
\Psi_{2}(\tau)=\left(\frac{\beta}{\beta+\tau}\right)^{\mu}
$$


Thus the assumption of the dependence of transition rate $\gamma_{2}(\tau)$ on the residence time like (24) leads to a power-law probability density function of residence time inside spines

$$
\psi_{2}(\tau)=\frac{d \Psi_{2}}{d \tau}=\frac{\mu}{\beta}\left(\frac{\beta}{\beta+\tau}\right)^{\mu+1} .
$$

The experimental evidence to support the hypothesis of a power-law distribution like (25) with $\mu<1$ is the subdiffusive transport of particles in a spiny dendrite [8, 16]. In what follows we will use this distribution to derive the fractional equations for the densities of particles.

\section{NON-MARKOVIAN TWO-STATE MODEL}

The aim of this section is to set up a non-Markovian model for the transport and reactions of particles in spiny dendrites by eliminating the residence time variable $\tau$. Let us denote the densities of particles just arriving in a dendrite and spines at point $x$ at time $t$ by $j_{1}(x, t)$ and $j_{2}(x, t)$ respectively. Obviously

$$
j_{i}(x, t)=\xi_{i}(x, 0, t) \quad i=1,2 .
$$

Note that in the paper [16] $j_{1}(x, t)$ denotes the number of particles arriving at point $x$ inside dendrite at time $t$ through a single spine stem (not a population of spines considered here) and $j_{2}(x, t)$ is the number of particles arriving at point $x$ in a single spine at time $t$.

To derive the balance equations for $j_{1}(x, t)$ and $j_{2}(x, t)$ we substitute (13)-(16) into the boundary conditions (11), (12) and use (10), (23). It gives the following equations for $j_{1}(x, t)$ and $j_{2}(x, t)$

$$
\begin{aligned}
j_{1}(x, t)= & \int_{0}^{t} \psi_{2}(\tau) j_{2}(x, t-\tau) e^{-\int_{t-\tau}^{t} r_{2}^{-}\left(n_{2}(x, s)\right) d s} d \tau \\
& +n_{2}^{0}(x) e^{-\int_{0}^{t} r_{2}^{-}\left(n_{2}(x, s)\right) d s} \int_{t}^{\infty} \psi_{2}(\tau) \Psi_{2}^{-1}(\tau-t) f_{2}(\tau-t \mid x) d \tau \\
j_{2}(x, t)= & \int_{0}^{t} \int_{\mathbb{R}} \psi_{1}(\tau) j_{1}(z, t-\tau) p(x-z, \tau) d z d \tau \\
& +\int_{t}^{\infty} \int_{\mathbb{R}} \psi_{1}(\tau) \Psi_{1}^{-1}(\tau-t) n_{1}^{0}(z) f_{1}(\tau-t \mid z) p(x-z, \tau) d z d \tau
\end{aligned}
$$

Balance equations for $n_{1}(x, t)$ and $n_{2}(x, t)$ can be found by substitution of (13)-(16) and (10) into (7)

$$
\begin{aligned}
n_{1}(x, t)= & \left.\int_{0}^{t} \int_{\mathbb{R}} \Psi_{1}(\tau) j_{1}(z, t-\tau) p(x-z, \tau)\right) d z d \tau \\
& \cdot+\int_{t}^{\infty} \int_{\mathbb{R}} \Psi_{1}(\tau) \Psi_{1}^{-1}(\tau-t) n_{1}^{0}(z) f_{1}(\tau-t \mid z) p(x-z, \tau) d z d \tau \\
n_{2}(x, t)= & \int_{0}^{t} \Psi_{2}(\tau) j_{2}(x, t-\tau) e^{-\int_{t-\tau}^{t} r_{2}^{-}\left(n_{2}(x, s)\right) d s} d \tau \\
& +n_{2}^{0}(x) e^{-\int_{0}^{t} r_{2}^{-}\left(n_{2}(x, s)\right) d s} \int_{t}^{\infty} \Psi_{2}(\tau) \Psi_{2}^{-1}(\tau-t) f_{2}(\tau-t \mid x) d \tau .
\end{aligned}
$$

Note that similar equations have been formulated (not derived) in [16]. For nonhomogeneous case such as (6) instead of $p(x-z, \tau)$ we should use $p(x, \tau \mid z)$. In what follows we use the $\delta$-function for residence time distribution at $t=0$ :

$$
f_{i}(\tau \mid x)=\delta(\tau) \quad i=1,2
$$

It corresponds to the case when the residence time of all particles at $t=0$ equals to zero. Substitution of (31) into (27)-(30) and rearrangement of the integration variables, $t-t^{\prime}=\tau$, give

$$
\begin{aligned}
j_{1}(x, t)= & \int_{0}^{t} \psi_{2}\left(t-t^{\prime}\right) j_{2}\left(x, t^{\prime}\right) e^{-\int_{t^{\prime}}^{t} r_{2}^{-}\left(n_{2}(x, s)\right) d s} d t^{\prime} \\
& +n_{2}^{0}(x) \psi_{2}(t) e^{-\int_{0}^{t} r_{2}^{-}\left(n_{2}(x, s)\right) d s},
\end{aligned}
$$




$$
\begin{aligned}
j_{2}(x, t)= & \int_{0}^{t} \int_{\mathbb{R}} \psi_{1}\left(t-t^{\prime}\right) j_{1}\left(z, t^{\prime}\right) p\left(x-z, t-t^{\prime}\right) d z d t^{\prime} \\
& +\psi_{1}(t) \int_{\mathbb{R}} n_{1}^{0}(z) p(x-z, t) d z .
\end{aligned}
$$

The balance equations for the densities $n_{i}(x, t)$ are

$$
\begin{aligned}
n_{1}(x, t)= & \left.\int_{0}^{t} \int_{\mathbb{R}} \Psi_{1}\left(t-t^{\prime}\right) j_{1}\left(z, t^{\prime}\right) p\left(x-z, t-t^{\prime}\right)\right) d z d t^{\prime} \\
& +\Psi_{1}(t) \int_{\mathbb{R}} n_{1}^{0}(z) p(x-z, t) d z, \\
n_{2}(x, t)= & \int_{0}^{t} \Psi_{2}\left(t-t^{\prime}\right) j_{2}\left(x, t^{\prime}\right) e^{-\int_{t^{\prime}}^{t} r_{2}^{-}\left(n_{2}(x, s)\right) d s} d t^{\prime} \\
& +n_{2}^{0}(x) \Psi_{2}(t) e^{-\int_{0}^{t} r_{2}^{-}\left(n_{2}(x, s)\right) d s .}
\end{aligned}
$$

To obtain the nonlinear Master equations for $n_{1}(x, t)$ and $n_{2}(x, t)$ we differentiate the densities given by (34) and (35) with respect to time $t$

$$
\begin{aligned}
\frac{\partial n_{1}}{\partial t}= & \left.\int_{\mathbb{R}} \Psi_{1}(0) j_{1}(z, t) p(x-z, 0) d z-\int_{0}^{t} \int_{\mathbb{R}} \psi_{1}\left(t-t^{\prime}\right) j_{1}\left(z, t^{\prime}\right) p\left(x-z, t-t^{\prime}\right)\right) d z d t^{\prime} \\
& +\int_{0}^{t} \int_{\mathbb{R}} \Psi_{1}\left(t-t^{\prime}\right) j_{1}\left(z, t^{\prime}\right) \frac{\partial p\left(x-z, t-t^{\prime}\right)}{\partial t} d z d t^{\prime}-\psi_{1}(t) \int_{\mathbb{R}} n_{1}^{0}(z) p(x-z, t) d z \\
& +\Psi_{1}(t) \int_{\mathbb{R}} n_{1}^{0}(z) \frac{\partial p(x-z, t)}{\partial t} d z
\end{aligned}
$$

and

$$
\begin{aligned}
\frac{\partial n_{2}}{\partial t}= & j_{2}(x, t)-\int_{0}^{t} \psi_{2}\left(t-t^{\prime}\right) j_{2}\left(x, t^{\prime}\right) e^{-\int_{t^{\prime}}^{t} r_{2}^{-}\left(n_{2}(x, s)\right) d s} d t^{\prime}-n_{2}^{0}(x) \psi_{2}(t) e^{-\int_{0}^{t} r_{2}^{-}\left(n_{2}(x, s)\right) d s} \\
& -r_{2}^{-}\left(n_{2}(x, t)\right)\left[\int_{0}^{t} \Psi_{2}\left(t-t^{\prime}\right) j_{2}\left(x, t^{\prime}\right) e^{-\int_{t^{\prime}}^{t} r_{2}^{-}\left(n_{2}(x, s)\right) d s} d t^{\prime}+n_{2}^{0}(x) \Psi_{2}(t) e^{-\int_{0}^{t} r_{2}^{-}\left(n_{2}(x, s)\right) d s}\right] .
\end{aligned}
$$

Using (32)-(35) together with (17), (18) and $\Psi_{1}(0)=1$, we can rewrite the last system of equations in a compact form

$$
\begin{gathered}
\frac{\partial n_{1}}{\partial t}=L_{x} n_{1}+j_{1}(x, t)-j_{2}(x, t), \\
\frac{\partial n_{2}}{\partial t}=j_{2}(x, t)-j_{1}(x, t)-r_{2}^{-}\left(n_{2}\right) n_{2},
\end{gathered}
$$

where the densities $j_{1}(x, t)$ and $j_{2}(x, t)$ describe the flux of particles between a population of spines and a parent dendrite. Now we need to express $j_{1}(x, t)$ and $j_{2}(x, t)$ in terms of $n_{2}(x, t)$ and $n_{1}(x, t)$ respectively. We obtain (Appendix C)

$$
\begin{gathered}
j_{1}(x, t)=\int_{0}^{t} K_{2}\left(t-t^{\prime}\right) n_{2}\left(x, t^{\prime}\right) e^{-\int_{t^{\prime}}^{t} r_{2}^{-}\left(n_{2}(x, s)\right) d s} d t^{\prime}, \\
j_{2}(x, t)=\int_{0}^{t} \int_{\mathbb{R}} K_{1}\left(t-t^{\prime}\right) p\left(x-z, t-t^{\prime}\right) n_{1}\left(z, t^{\prime}\right) d z d t^{\prime},
\end{gathered}
$$

where $K_{i}(t)$ is the memory kernel defined by

$$
\tilde{K}_{i}(s)=\frac{\tilde{\psi}_{i}(s)}{\tilde{\Psi}_{i}(s)} \quad i=1,2 .
$$


The nonlinear Master equations (36) and (37) together with interaction terms (38) and (39) is a generalization of a linear system of equations obtained in [16]. In contrast to the classical Markovian model (3) and (44), the spinesdendrite interaction terms $j_{1}(x, t)$ and $j_{2}(x, t)$ are not-local in time and space. The density $j_{1}(x, t)$ describes the average flux of particles from a population of spines through spine necks into parent dendrite. The characteristic feature of this flux is that it depends on chemical reactions through the exponential term $e^{-\int_{t^{\prime}}^{t} r_{2}^{-}\left(n_{2}(x, s)\right) d s}$. It means that one can not separate the flux of particles from spines to a dendrite from the chemical reactions inside the spines. Similar effects have been found for the reaction-transport systems in [21, 27, 29]. This phenomenon does not exist in the Markovian case for which the memory kernel is delta-function

$$
K_{i}(t)=\gamma_{i} \delta(t)
$$

since $\tilde{K}_{i}(s)=\gamma_{i}$. In this case it follows from (38), (39) and (41) that the fluxes of particles are local:

$$
j_{1}(x, t)=\gamma_{2} n_{2}(x, t), \quad j_{2}(x, t)=\gamma_{1} n_{1}(x, t) .
$$

Note that the flux of particles from dendrite to spines (39) depends on the transport process inside dendrite.

\section{ANOMALOUS ADVECTION}

Consider the case when there is no reaction inside the spines: $r_{2}^{-}\left(n_{2}\right)=0$. It has been found [16] that if the particle inside dendrite performs a Brownian motion and the residence time's PDF $\psi_{2}(\tau)$ behaves like

$$
\psi_{2}(\tau) \sim\left(\frac{\tau_{2}}{\tau}\right)^{1+\mu}, \quad \mu<1
$$

as $\tau \rightarrow \infty$, the mean squared displacement, $\left\langle x^{2}(t)\right\rangle$, exhibits a subdiffusive behavior $t^{\mu}$. The reason for this anomalous diffusion is that the population of spines acts as a trap of particles [8].

The aim of this section is to show that if the particles move inside a dendrite with constant velocity $v$, the mean particle's position $\langle x(t)\rangle$ increases as $t^{\mu}$ (anomalous advection). For a constant velocity $v$, the transport operator takes the form

$$
L_{x} n_{1}=-v \frac{\partial n_{1}}{\partial x}
$$

First we find the Laplace transform of $\langle x(t)\rangle$

$$
\langle x(s)\rangle=-i\left(\frac{d n(k, s)}{d k}\right)_{k=0},
$$

where $n(k, s)=n_{1}(k, s)+n_{2}(k, s)$ is the Fourier-Laplace transform of the total density of particles $n=n_{1}+n_{2}$ :

$$
n(k, s)=\int_{\mathbb{R}} \int_{0}^{\infty} e^{i k x-s t} n(x, t) d t d x .
$$

Applying the Fourier-Laplace transform to (36)-(39) with $r_{2}^{-}\left(n_{2}\right)=0$, we obtain

$$
n(k, s)=\frac{n_{1}^{0}(k)\left(\tilde{\Psi}_{1}(s-\varphi(k))+\tilde{\psi}_{1}(s-\varphi(k)) \tilde{\Psi}_{2}(s)\right)+n_{2}^{0}(k)\left(\tilde{\Psi}_{2}(s)+\tilde{\psi}_{2}(s) \tilde{\Psi}_{1}(s-\varphi(k))\right)}{1-\tilde{\psi}_{2}(s) \tilde{\psi}_{1}(s-\varphi(k))} .
$$

Consider the particular case when the initial density of particles inside spines is zero $\left(n_{2}^{0}(k)=0\right)$ and the initial density inside a dendrite is delta-function: $n_{1}(x, 0)=\delta(x)\left(n_{1}^{0}(k)=1\right)$. Then

$$
n(k, s)=\frac{\tilde{\Psi}_{1}(s-i k v)+\tilde{\psi}_{1}(s-i k v) \tilde{\Psi}_{2}(s)}{1-\tilde{\psi}_{2}(s) \tilde{\psi}_{1}(s-i k v)} .
$$

since $\varphi(k)=i k v$ for the transport operator defined by (44). We assume that the residence time's PDFs $\psi_{1}(\tau)$ is exponential:

$$
\psi_{1}(\tau)=\gamma_{1} e^{-\gamma_{1} \tau}
$$


with the Laplace transform

$$
\tilde{\psi}_{1}(s)=\frac{\gamma_{1}}{\gamma_{1}+s}
$$

and $\tilde{\Psi}_{1}(s)=1 /\left(\gamma_{1}+s\right)$. The Laplace transform $\tilde{\psi}_{2}(s)$ corresponding to (43) can be approximated by

$$
\tilde{\psi}_{2}(s) \sim 1-\left(\tau_{2} s\right)^{\mu}, \quad \mu<1
$$

for small $s[9] ; \tau_{2}$ is a parameter with units of time. The mean waiting time $\langle\tau\rangle=\int_{0}^{\infty} \tau \psi_{2}(\tau) d \tau$ is infinite in this case. In the limit $s \rightarrow 0$, we find from (48), (49) and (50) that

$$
n(k, s) \sim \frac{\gamma_{1} \tau_{2}^{\mu}}{s^{1-\mu}\left(-i k v+\gamma_{1} \tau_{2}^{\mu} s^{\mu}\right)} .
$$

By using (45) we find

$$
\langle x(s)\rangle \sim \frac{v}{s^{1+\mu} \gamma_{1} \tau_{2}^{\mu}} .
$$

Thus the average position of particle is

$$
\langle x(t)\rangle \sim \frac{v}{\Gamma(1+\mu) \gamma_{1} \tau_{2}^{\mu}} t^{\mu}, \quad \mu<1
$$

which is sublinear. This anomalous advection reflects the memory effect corresponding to the slow movement of particles in spiny dendrites due to the power law of the residence time distribution for spines (433).

\section{EVOLUTION EQUATION FOR TOTAL DENSITY IN THE LONG-TIME LIMIT}

In this section we derive a governing equation for the total density of particles $n(x, t)=n_{1}(x, t)+n_{2}(x, t)$ in the limit $t \rightarrow \infty$. We consider the case when initially all particles are inside dendrite: $n(x, 0)=n_{1}^{0}(x)$ and $n_{2}^{0}(x)=0$. By using $\tilde{\psi}_{1}(s+i k v) / \tilde{\Psi}_{1}(s+i k v)=\gamma_{1}$, we rearrange (47) as

$$
s n(k, s)-n_{1}^{0}(x)=\frac{\varphi(k) n(k, s)}{1+\gamma_{1} \tilde{\Psi}_{2}(s)} .
$$

Inverse Fourier-Laplace transform gives

$$
\frac{\partial n}{\partial t}=\int_{0}^{t} G(t-s) L_{x} n(x, s) d s,
$$

where the memory kernel $G$ defined by its Laplace transform

$$
\tilde{G}(s)=-\frac{1}{1+\gamma_{1} \tilde{\Psi}_{2}(s)} .
$$

Note that this kernel is different from the standard one in CTRW models (40).

Standard advection-diffusion equation. First let us consider the case when the residence time PDF for spines is

$$
\psi_{2}(\tau)=\gamma_{2} e^{-\gamma_{2} \tau}
$$

with $\tilde{\Psi}_{2}(s)=1 /\left(\gamma_{2}+s\right)$. We assume that

$$
\varphi(k)=i v k-D k^{2} .
$$

In the long-time limit $t \rightarrow \infty(s \rightarrow 0)$, we obtain from (52) the governing equation for the total density

$$
\frac{\partial n}{\partial t}+v^{*} \frac{\partial n}{\partial x}=D^{*} \frac{\partial^{2} n}{\partial x^{2}}
$$


where

$$
v^{*}=\frac{\gamma_{2}}{\gamma_{1}+\gamma_{2}} v, \quad D^{*}=\frac{\gamma_{2}}{\gamma_{1}+\gamma_{2}} D
$$

Note that Eq. (57) is valid for any residence times PDF's $\psi_{1}(\tau)$ and $\psi_{2}(\tau)$ with finite mean residence times $\left\langle\tau_{1}\right\rangle$ and $<\tau_{2}>$ respectively. The leading order behavior of the Laplace transforms $\tilde{\psi}_{1}(s)$ and $\tilde{\psi}_{2}(s)$ as $s \rightarrow 0$ is

$$
\tilde{\psi}_{i}(s) \sim 1-<\tau_{i}>s, \quad i=1,2 .
$$

Then the effective velocity $v^{*}$ and diffusivity $D^{*}$ are

$$
v^{*}=\frac{<\tau_{1}>}{<\tau_{1}>+<\tau_{2}>} v, \quad D^{*}=\frac{<\tau_{1}>}{<\tau_{1}>+<\tau_{2}>} D .
$$

Anomalous advection-diffusion equation. Now we consider the case when the residence time PDF $\psi_{2}(\tau)$ behaves like (43). The infinite mean residence time leads to the anomalous transport of particles along spiny dendrite. By using (50) we obtain the Laplace transform of the survival probability

$$
\tilde{\Psi}_{2}(s)=\frac{1-\tilde{\psi}_{2}(s)}{s}=\frac{\tau_{2}^{\mu}}{s^{1-\mu}} .
$$

Substitution of (60) into (52) gives

$$
\gamma_{1} \tau_{2}^{\mu}\left(s^{\mu} n(k, s)-s^{\mu-1} n_{1}^{0}(x)\right)=\varphi(k) n(k, s),
$$

where $\varphi(k)$ is defined by (56). We apply the Fourier-Laplace transform inversion and obtain the fractional advectiondiffusion equation

$$
\gamma_{1} \tau_{2}^{\mu} \frac{\partial^{\mu} n}{\partial t^{\mu}}+v \frac{\partial n}{\partial x}=D \frac{\partial^{2} n}{\partial x^{2}},
$$

where

$$
\frac{\partial^{\mu} n}{\partial t^{\mu}}=\frac{1}{\Gamma(1-\mu)} \frac{\partial}{\partial t} \int_{0}^{t} \frac{n(x, \tau)}{(t-\tau)^{\mu}} d \tau-\frac{n_{1}^{0}(x)}{\Gamma(1-\mu) t^{\mu}}
$$

is the Caputo fractional derivative [17. Note that the fractional Nernst-Planck equations for ions' movement have been suggested in [10]. In general, the velocity of particles $v$ is not constant since the electric force acting on particles (ions) depends on the density of particles. This dependence is described by the Poisson-Nernst-Planck (PNP) equations. The challenge is to derive the fractional analog of these equations from the mesoscopic model. Obviously the fractional equation (61) is just a first step in this direction.

In fact our model could be used for other applications involving two states: active phase and a quiescent (immobile) phase. The classical Markovian switching models involve the Poisson processes with exponential distribution of residence times (see, for example, [32]). The non-Markovian switching model developed here can be useful in biological modeling 32 35], front propagation in systems with aging and random switching in velocities [36], largescale transport of solutes in fractured rock involving mobile/immobile transport with power law memory functions [37], directed intermittent search on a tree network [38].

\section{CONCLUSIONS}

The aim was to give a mesoscopic description of the anomalous transport and reactions of particles in spiny dendrites. We extended two-state linear model presented in [16] and derived the nonlinear Master equations for the average densities of particles inside spines and a parent dendrite. As a starting point we used the Markovian model with an assumption that the transition probabilities depend on the residence time variable. Motivated by the experiments [8] on anomalous transport of particles along the dendrite we assumed that the longer a particle survives inside a spine, the smaller becomes the transition probability from spine to dendrite. By using power-law residence time distributions for spines we found that if particles move inside a dendrite with constant velocity $v$, the mean particle's position $\langle x(t)\rangle$ increases as $t^{\mu}$ with $\mu<1$ (anomalous subadvection). Fractional advection-diffusion equation for the total density of particles was derived. We showed that the interaction terms describing the flux of 
particles between spines and parent dendrite are not-local in time and space. In particular the average flux of particles from a population of spines through spine necks into parent dendrite depends on chemical reactions in spines. It means that one can not separate the flux of particles from spines to dendrite from the chemical reactions. This phenomenon does not exist in the Markovian case. The flux of particles from dendrite to spines is found to depend on the transport process inside dendrite. These results might have a significant implication for nerve cell signalling. The main reason for this is that the effective electro-diffusion of ions inside spiny dendrite can not be separated form reactions inside spines. An interesting issue is whether this effect has any significant effect on the transport of $C a^{2+}$. One can conclude that the exponential factor $\exp \left(-\int_{t^{\prime}}^{t} r_{2}^{-}\left(n_{2}(x, s)\right) d s\right)$ in (38) explains the effect of limited diffusion of $C a^{2+}$ along dendrites observed in experiments 8]. The derivation of anomalous cable theory from the nonlinear Master equations (36) and (37) together with interaction terms (38) and (39) will be the subject of future work.

\section{Acknowledgment}

This research was supported by the grant $\Gamma \mathrm{K}$ N02-740-11-5172 "Anomalous transport in biological and chemical systems".

[1] E. Nimchinsky, B. Sabatini, K. Svoboda K, Ann. Rev. Physiol. 64, 31353 (2002).

[2] M. Segal, Nature Rev. Neurosci. 6, 277 (2005).

[3] S. M. Baer and J. Rinzel, J. Neurophysiol. 65, 874 (1991).

[4] H-Y. Wu and S. M. Baer, J. Math. Biol 36, 569 (1998).

[5] S. Coombes and P.C. Bressloff, SIAM J Appl. Math. 61, 432 (2000); Phys. Rev. Lett. 91, 028102 (2002).

[6] B.A. Earnshaw and P. C. Bressloff, J. Comput. Neurosci. 25, 366 (2008).

[7] Y. Timofeeva, Physica D 239, 494 (2010).

[8] F. Santamaria, S. Wils, E. De Schutter, and G. J. Augustine, Neuron 52, 635 (2006).

[9] R. Metzler and J. Klafter, Phys. Rep. 339, 1 (2000).

[10] B. I. Henry, T. A. M. Langlands, S. L. Wearne, Phys. Rev. Lett. 100, 128103 (2008); T. A. M. Langlands, B. I. Henry,, S. L. Wearne, J. Math. Biology 59, 761 (2009).

[11] B. L. Sabatini, M. Maravall, and K. Svoboda, Curr. Opin. Neurobiol. 11, 349 (2001); B. L. Bloodgood, B. L. Sabatini, Science 310, 866 (2005).

[12] Z. Schuss, A. Singer, and D. Holcman, PNAS 104, 16098 (2007).

[13] A. M. Berezhkovskii, A. V. Barzykin and V. Yu. Zitserman, J. Chem. Phys. 130, 245104 (2009).

[14] E. Korkotian and M. Segal, Cell Calcium 40, 441 (2006).

[15] L. Dagdug, A. M. Berezhkovskii, Y. A. Makhnovskii, and V. Yu. Zitserman, J. Chem. Phys. 127224712 (2007).

[16] S. Fedotov and V. Méndez, Phys. Rev. Lett. 101, 218102 (2008).

[17] V. Méndez, S. Fedotov and W. Horsthemke, Reaction-Transport Systems (Springer, Berlin 2010).

[18] D. R. Cox and H. D. Miller, The Theory of Stochastic Processes (Methuen, London, 1965).

[19] N. G. van Kampen, Physica A 96, 435 (1979).

[20] M. O. Vlad and J. Ross, Phys. Rev. E 66, 061908 (2002).

[21] A. Yadav and W. Horsthemke, Phys. Rev. E 74, 066118 (2006).

[22] Y. Nec and A. A. Nepomnyashchy, J. Phys. A: Math. Theor. 4014687 (2007).

[23] H. Wang and H. Qian, J. Math. Physics 48, 013303 (2007); H. Qian and H. Wang, Europhys. Lett. 76, 15 (2006).

[24] I. Goychuk, P. Hanggi. Phys. Rev. Lett., 91070601 (2003).

[25] A. I. Shushin, Phys. Rev. E, 64051108 (2001); D. Campos, S. Fedotov, and V. Mendez, Phys. Rev. E 77, 061130 (2008).

[26] I. Goychuk, P. Hanggi, Phys. Rev. E, 70051915 (2004).

[27] S. Fedotov, A. Iomin, Phys. Rev. Lett., 98118101 (2007); Phys. Rev. E 77, 031911 (2008).

[28] S. Fedotov, G. N. Milstein and M. V. Tretyakov, J. Phys. A: Math. Theor. 40, 5769 (2007); V. P. Shkilev, J. Experimental and Theoretical Physics, 107, 892 (2008); M.A.A. da Silva, J.C. Cressoni, G.M. Viswanathan, Physica A 364,70 (2006).

[29] I. M. Sokolov, M. G. Schmidt, F. Sagues, Phys. Rev. E 73, 031102 (2006); F. Sagues, V. P. Shkilev, and I. M. Sokolov, Phys. Rev. E 77, 032102 (2008); B. I. Henry, T. A. M. Langlands, and S. L. Wearne, Phys. Rev. E 74, 031116 (2006).

[30] S. Fedotov, Phys. Rev. E 81, 011117 (2010).

[31] J. D. Murry, Mathematical Biology (Springer, Berlin 2003).

[32] L. Bilinsky, K.P. Hadeler. J. Biol. Dynamics 3, 751 (2009); K.P. Hadeler, T. Hillen, M. Lewis, Biological Modeling with Quiescent Phases. Chapter 6 in: C. Cosner, S. Cantrell, S. Ruan, Spatial Ecology, Taylor and Francis 2009.

[33] D. V. Nicolau Jr., J. F. Hancock, and K. Burrage, Biophysics Journal 92, 1975 (2007); B. A. Earnshaw and P. C. Bressloff, J. Comp. Neuroscience, 28, 77 (2010).

[34] V. Méndez, D. Campos and A. W. Sheppard. Bull. Math. Biol. 71, 1727 (2009).

[35] J. Fort and V. Méndez, Phys. Rev. Lett. 89, 178101 (2001). 
[36] D. Campos and V. Méndez, Phys. Rev. E 80, 021133 (2009); J. Phys. A: Math. Theor. 42, 075003 (2009).

[37] R. Schumer, D. A. Benson, M.M. Meerschaert and B. Baeumer, Water Resources Research, 391, 296 (2003); D.M. Reeves, D.A. Benson, M.M. Meerschaert, Water Resources Research, 44 W08405 (2008); D.A. Benson, M.M. Meerschaert, Advances in Water Resources, 32532 (2009).

[38] J. M. Newby and P. C. Bressloff, Phys. Rev. E 80, 021913 (2009).

\section{APPENDIX A}

To derive the system of equations (8) and (9), we start with the balance of particles at the point $x$ For a dendrite

$$
\xi_{1}(x, \tau+h, t+h)=\left(1-\gamma_{1}(\tau) h-\lambda(x) h\right) \xi_{1}(x, \tau, t)+h \int_{\mathbb{R}} \lambda(z) \xi_{2}(z, \tau, t) w(x-z) d z+o(h) .
$$

For spines

$$
\xi_{2}(x, \tau+h, t+h)=\left(1-\gamma_{2}(\tau) h\right) \xi_{2}(x, \tau, t)-r_{2}^{-}\left(n_{2}\right) \xi_{2}(x, \tau, t) h+o(h) .
$$

The first equation states that the density of particles $\xi_{1}(x, \tau+h, t+h)$ at point $x$ with the residence time $\tau+h$ inside dendrite at time $t+h$ is the sum of the density of particles with the residence time $\tau$ at time $t$ multiplied by the survival probability $1-\gamma_{1}(\tau) h-\lambda(x) h$ and the density of particles that jump from different positions $z$. The jump length $x-z$ is distributed according to the dispersal kernel or jump length PDF $w(x-z)$. The second equation describes the balance of particles inside spines. The factor $1-\gamma_{2}(\tau) h$ in the RHS of (63) is the probability that the particles make no transition from spines to dendrite during small time period $(\tau, \tau+h]$. The last term describes the decrease of the density $\xi_{2}$ due to the chemical reaction with the rate $r_{2}^{-}\left(n_{2}\right)$ that depends of the local density of particles $n_{2}$. The advantage to have a Markovian model is that the balance of particles during time $(t, t+h]$ is independent of what happened during the previous time interval $(0, t]$.

Subtracting $\xi_{1}(x, \tau, t)$ from both sides of the balance equation (62) and subtracting $\xi_{2}(x, \tau, t)$ from (63), dividing by $h$, and letting $h \rightarrow 0$, we obtain the mesoscopic system of reaction-transport equations (8) and (9) with the transport operator $L_{x}$ given by (5) .

\section{APPENDIX B}

First we find the solution to (8). We denote the Fourier transform of $\xi_{1}(x, \tau, t)$ by $\hat{\xi}_{1}(k, \tau, t)$. Applying the Fourier transform and convolution theorem to (8), we obtain

$$
\frac{\partial \hat{\xi}_{1}}{\partial t}+\frac{\partial \hat{\xi}_{1}}{\partial \tau}=\varphi(k) \hat{\xi}_{1}-\gamma_{1}(\tau) \hat{\xi}_{1}
$$

where $\varphi(k)$ is the symbol of the pseudo-differential operator $L_{x}$. It means that the Fourier transform of $L_{x} \xi_{1}$ can be written as

$$
\mathcal{F}\left[L_{x} \xi_{1}(x, \tau, t)\right]=\varphi(k) \hat{\xi}_{1}(k, \tau, t)
$$

In particular, for the advection-diffusion operator (6) with the constant advection velocity $v$, we have

$$
\varphi(k)=i v k-D k^{2}
$$

For the operator (5) with the constant transition rate $\lambda$, we obtain

$$
\varphi(k)=\lambda(\hat{w}(k)-1),
$$

where $\varphi(k)$ is the characteristic exponent of compound Poisson process with intensity $\lambda$ and $\hat{w}(k)$ is the Fourier transform of jump PDF $w(z)$ [17].

The characteristics of PDE (64) are determined by the equation $d \tau / d t=1$. Along the straight lines (characteristics) $\tau(t)=t-t_{0}$ and $\tau(t)=t+\tau_{0}$, a partial differential equation (PDE) (64) is reduced to

$$
\frac{d \hat{\xi}_{1}(k, \tau(t), t)}{d t}=\varphi(k) \hat{\xi}_{1}-\gamma_{1}(\tau(t)) \hat{\xi}_{1}
$$


This equation has the solution

$$
\hat{\xi}_{1}(k, \tau(t), t)=\hat{\xi}_{1}\left(k, 0, t_{0}\right) e^{\varphi(k)\left(t-t_{0}\right)-\int_{t_{0}}^{t} \gamma_{1}(\tau(s)) d s} \quad \text { for } \quad \tau(t)=t-t_{0}
$$

and

$$
\hat{\xi}_{1}(k, \tau(t), t)=\hat{\xi}_{1}\left(k, \tau_{0}, 0\right) e^{\varphi(k) t-\int_{0}^{t} \gamma_{1}(\tau(s)) d s} \quad \text { for } \quad \tau(t)=t+\tau_{0} .
$$

Using the inverse Fourier transform and the facts that

$$
\begin{aligned}
& \mathcal{F}^{-1}\left[\hat{\xi}_{1}(k, 0, t-\tau) e^{\varphi(k) \tau}\right]=\int_{\mathbb{R}} \xi_{1}(z, 0, t-\tau) p(x-z, \tau) d z \quad \text { for } \quad \tau<t, \\
& \mathcal{F}^{-1}\left[\hat{\xi}_{1}(k, \tau-t, 0) e^{\varphi(k) t}\right]=\int_{\mathbb{R}} \xi_{1}(z, \tau-t, 0) p(x-z, t) d z \quad \text { for } \quad \tau>t,
\end{aligned}
$$

we obtain (13) and (14):

$$
\begin{aligned}
& \xi_{1}(x, \tau, t)=e^{-\int_{0}^{\tau} \gamma_{1}(s) d s} \int_{\mathbb{R}} \xi_{1}(z, 0, t-\tau) p(x-z, \tau) d z \quad \text { for } \quad \tau<t, \\
& \xi_{1}(x, \tau, t)=e^{-\int_{\tau-t}^{\tau} \gamma_{1}(s) d s} \int_{\mathbb{R}} \xi_{1}(z, \tau-t, 0) p(x-z, t) d z \quad \text { for } \quad \tau>t,
\end{aligned}
$$

where $p(x, t)$ is the inverse Fourier transform of

$$
\hat{p}(k, t)=e^{\varphi(k) t} .
$$

In particular, $p(x, t)$ is the Green function for the Kolmogorov-Feller equation

$$
\frac{\partial p}{\partial t}=-\lambda p+\lambda \int_{\mathbb{R}} p(z, t) w(x-z) d z
$$

with the initial condition $p(x, 0)=\delta(x)$ and $\varphi(k)=\lambda(\hat{w}(k)-1)$.

The equation for the density $\xi_{2}(x, \tau, t)$

$$
\frac{\partial \xi_{2}}{\partial t}+\frac{\partial \xi_{2}}{\partial \tau}=-\gamma_{2}(\tau) \xi_{2}-r_{2}^{-}\left(n_{2}\right) \xi_{2}
$$

can be solved by the method of characteristics in the same way. One can obtain

$$
\xi_{2}(x, \tau(t), t)=\xi_{2}\left(x, 0, t_{0}\right) e^{-\int_{t_{0}}^{t} \gamma_{2}(\tau(s)) d s-\int_{t_{0}}^{t} r_{2}^{-}\left(n_{2}(x, s)\right) d s} \quad \text { for } \quad \tau(t)=t-t_{0}
$$

and

$$
\xi_{2}(x, \tau(t), t)=\xi_{2}\left(x, \tau_{0}, 0\right) e^{-\int_{0}^{t} \gamma_{2}(\tau(s)) d s-\int_{0}^{t} r_{2}^{-}\left(n_{2}(x, s)\right) d s} \quad \text { for } \quad \tau(t)=t+\tau_{0} .
$$

These solutions can be rewritten in the forms given in (15) and (16).

\section{APPENDIX C.}

Multiplying (32) and (35) by $e^{\int_{0}^{t} r_{2}^{-}\left(n_{2}(x, s)\right) d s}$ and taking the Laplace transform $\mathcal{L}\{f\}$, we obtain

$$
\begin{aligned}
\mathcal{L}\left\{j_{1}(x, t) e^{\int_{0}^{t} r_{2}^{-}\left(n_{2}(x, s)\right) d s}\right\} & =\left[n_{2}^{0}(x)+\mathcal{L}\left\{j_{2}(x, t) e^{\int_{0}^{t} r_{2}^{-}\left(n_{2}(x, s)\right) d s}\right\}\right] \tilde{\psi}_{2}(s), \\
\mathcal{L}\left\{n_{2}(x, t) e^{\int_{0}^{t} r_{2}^{-}\left(n_{2}(x, s)\right) d s}\right\} & =\left[n_{2}^{0}(x)+\mathcal{L}\left\{j_{2}(x, t) e^{\int_{0}^{t} r_{2}^{-}\left(n_{2}(x, s)\right) d s}\right\}\right] \tilde{\Psi}_{2}(s) .
\end{aligned}
$$


Then

$$
\mathcal{L}\left\{j_{1}(x, t) e^{\int_{0}^{t} r_{2}^{-}\left(n_{2}(x, s)\right) d s}\right\}=\mathcal{L}\left\{n_{2}(x, t) e^{\int_{0}^{t} r_{2}^{-}\left(n_{2}(x, s)\right) d s}\right\} \frac{\tilde{\psi}_{2}(s)}{\tilde{\Psi}_{2}(s)} .
$$

Inverse Laplace transform gives

$$
j_{1}(x, t) e^{\int_{0}^{t} r_{2}^{-}\left(n_{2}(x, s)\right) d s}=\int_{0}^{t} K_{2}\left(t-t^{\prime}\right) n_{2}\left(x, t^{\prime}\right) e^{\int_{0}^{t^{\prime}} r_{2}^{-}\left(n_{2}(x, s)\right) d s} d t^{\prime},
$$

where $K_{2}(t)$ is the memory kernel defined by

$$
\tilde{K}_{2}(s)=\frac{\tilde{\psi}_{2}(s)}{\tilde{\Psi}_{2}(s)}
$$

From (71), we get (38).

Now let us find the expression for $j_{2}(x, t)$ in terms of $n_{1}(x, t)$. Taking the Fourier-Laplace transform of (33) and (34), we obtain

$$
\begin{aligned}
& \tilde{j}_{2}(k, s)=\left(\tilde{j}_{1}(k, s)+\hat{n}_{1}^{0}(k)\right) \mathcal{L}\left\{\psi_{1}(t) \hat{p}(k, t)\right\}, \\
& \tilde{n}_{1}(k, s)=\left(\tilde{j}_{1}(k, s)+\hat{n}_{1}^{0}(k)\right) \mathcal{L}\left\{\Psi_{1}(t) \hat{p}(k, t)\right\} .
\end{aligned}
$$

Taking into account (69) and shift theorem, we find

$$
\mathcal{L}\left\{\psi_{1}(t) \hat{p}(k, t)\right\}=\tilde{\psi}_{1}(s-\varphi(k)), \quad \mathcal{L}\left\{\Psi_{1}(t) \hat{p}(k, t)\right\}=\tilde{\Psi}_{1}(s-\varphi(k)) .
$$

We have

$$
\tilde{j}_{2}(k, s)=\tilde{n}_{1}(k, s) \frac{\tilde{\psi}_{1}(s-\varphi(k))}{\tilde{\Psi}_{1}(s-\varphi(k))}=\tilde{n}_{1}(k, s) \tilde{K}_{1}(s-\varphi(k)) .
$$

Inverse Fourier-Laplace transform of (173) gives (39). 